\title{
Undiagnosed bipolar disorder in patients treated for major depression in primary care Jonathan Silva ${ }^{1}$ ${ }^{1}$ USF Balsa, Tavira, Portugal.
}

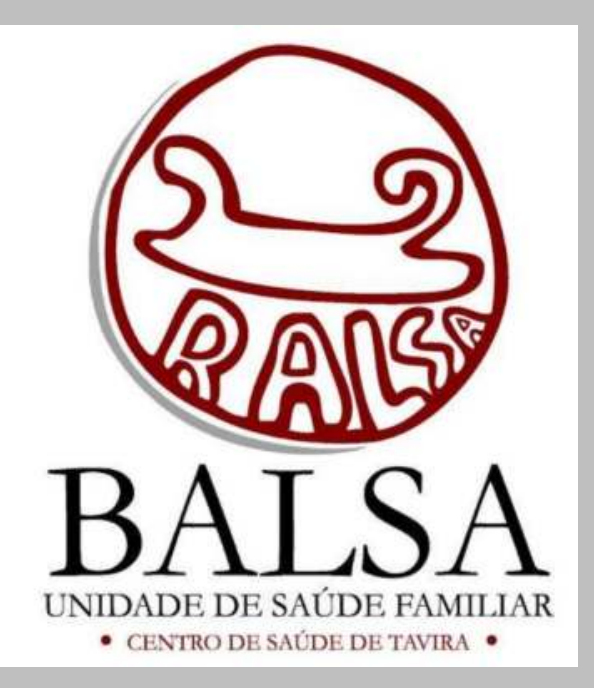

\section{BACKGROUND AND AIMS}

Early identification of Bipolar Disorder (BD), particularly in the depressive phase, can be challenging. For many BD patients a depressive episode is often the first mood syndrome at the onset of BD with multiple episodes of major depression usually occurring prior to the first episode of mania. Depressive phases occur more frequently than hypomanic or manic phases. As a result BD is frequently misdiagnosed as unipolar major depression disorder and may receive inappropriate treatment. Thus, early diagnosis is of crucial importance.

\section{MATERIALS AND METHODS}

Review of the literature published in the PubMed database, in the last 10 years, using the Mesh terms "bipolar disorder", "major depressive disorder", "misdiagnosis", "primary care" and "screening".

\section{RESULTS AND CONCLUSIONS}

Delay in diagnosis leads to a more severe form of BD. There are several demographic and clinical characteristics that are more commonly observed in bipolar disorder compared with unipolar depression which should be considered in order to early recognise BD (table 1).

\begin{tabular}{l} 
Table 1. Possible indicators of bipolar disorder in depressed \\
patients \\
Family history of bipolar disorder \\
\hline Earlier onset of illness (early 20's) \\
\hline Seasonality \\
\hline Numerous past episodes \\
\hline History of psychiatric hospitalization \\
\hline Mixed states \\
\hline Mood reactivity \\
\hline History of treatment-resistant depression \\
\hline Switching on antidepressants \\
\hline History of suicide attempt
\end{tabular}

Another strategy for improving diagnosis, may be the greater use of screening instruments. Several psychometric instruments have been developed to screen for BD. Evidence presented from the literature has shown that there are available screening tools for BD with reliable psychometric properties that may be useful in the primary care setting. The Hypomania Checklist-32 (HCL-32) is one of such self-report questionnaires, designed to screen for hypomania symptoms in patients with major depressive disorder.

While screening tools are not a replacement for diagnosis of a mental illness by a trained professional, they are helpful in assisting primary care practioners in early recognition. A positive screening may provide a reasonable basis for more comprehensive clinical assessment and give a reasonable foundation from wich a referral to psychiatry can be made. Integrating such screening tools with careful characterization of longitudinal course may provide the best strategy for diagnosis. 